\title{
X-Ray Emission from the First Quasars
}

\section{Citation}

Haiman, Zoltan, and Abraham Loeb. 1999. "X-Ray Emission from the First Quasars." The Astrophysical Journal 521 (1): L9-12. https://doi.org/10.1086/312177.

\section{Permanent link}

http://nrs.harvard.edu/urn-3:HUL.InstRepos:41393256

\section{Terms of Use}

This article was downloaded from Harvard University's DASH repository, and is made available under the terms and conditions applicable to Other Posted Material, as set forth at http:// nrs.harvard.edu/urn-3:HUL.InstRepos:dash.current.terms-of-use\#LAA

\section{Share Your Story}

The Harvard community has made this article openly available.

Please share how this access benefits you. Submit a story.

Accessibility 


\title{
X-RAY EMISSION FROM THE FIRST QUASARS
}

\author{
Zoltan Haiman \\ NASA/Fermilab Astrophysics Center, Fermi National Accelerator Laboratory, Batavia, IL 60510; zoltan@fnal.gov \\ AND \\ ABRAHAM LOEB \\ Harvard-Smithsonian Center for Astrophysics 60 Garden Street, Cambridge, MA 02138; aloeb@cfa.harvard.edu \\ Received 1999 April 26; accepted 1999 June 10; published 1999 July 16
}

\begin{abstract}
It is currently unknown whether the universe was reionized by quasars or stars at $z \gtrsim 5$. We point out that quasars can be best distinguished from stellar systems by their X-ray emission. Based on a simple hierarchical CDM model, we predict the number counts and X-ray fluxes of quasars at high redshifts. The model is consistent with available data on the luminosity function of high-redshift quasars in the optical and soft X-ray bands. The cumulative contribution of faint, undetected quasars in our model is consistent with the unresolved fraction of the X-ray background. We find that the Chandra X-ray Observatory might detect $\sim 10^{2}$ quasars from redshifts $z \geq 5$ per its $17^{\prime} \times 17^{\prime}$ field of view at the flux threshold of $\sim 2 \times 10^{-16} \mathrm{ergs} \mathrm{s}^{-1} \mathrm{~cm}^{-2}$. The redshifts of these faint point sources could be identified by follow-up infrared observations from the ground or with the Next Generation Space Telescope.
\end{abstract}

Subject headings: black hole physics — cosmology: theory — quasars: general

\section{INTRODUCTION}

Cold dark matter (CDM) cosmologies predict that the first baryonic objects appeared near the Jeans mass $\left(\sim 10^{6} M_{\odot}\right)$ at redshifts as high as $z \sim 30$, and larger objects assembled later (Haiman \& Loeb 1999b and references therein). It is natural to identify these objects as the sites where the first stars or quasar black holes formed. Observationally, bright quasars are currently detected out to $z \sim 5$ (Fan et al. 1999), and galaxies out to $z \sim 6.7$ (Chen et al. 1999; Weymann et al. 1998). Although the abundance of optically and radio bright quasars declines at $z \gtrsim 2.5$ (Schmidt, Schneider, \& Gunn 1995; Pei 1995; Shaver et al. 1996), simple models based on the PressSchechter (1974) formalism reproduce this decline and simultaneously predict a population of low-luminosity quasars that are too faint to be detected in current surveys (Haiman \& Loeb 1998; Haehnelt, Natarajan, \& Rees 1998). Preliminary evidence for such a population might already be indicated by the X-ray luminosity function (LF) of quasars recently measured by $\mathrm{RO}$ SAT (Miyaji, Hasinger, \& Schmidt 1998a). The X-ray data probe fainter quasars than the optical data do and has not revealed a decline in the abundance of high-redshift quasars as found in the optical (e.g., Schmidt et al. 1995).

The current census of high-redshift galaxies provides an estimate of the global evolution of the star formation rate (SFR) in the redshift range $0 \leq z \leq 5$ (Madau 1999). Nevertheless, there is still considerable uncertainty about the evolution of the SFR at the highest redshifts probed $(z \gtrsim 3)$. Recent sub-millimeter observations from SCUBA indicate the existence of a substantial population of dust-obscured star-forming galaxies that could raise the implied SFR at high redshift (Barger, Cowie, \& Sanders 1999, and references therein). This uncertainty is particularly important, since it leaves open the question of whether the observed population of stars produce sufficient ionizing radiation by $z \sim 5$ to reionize the intergalactic medium (IGM). The indication from the present data is that the SFR either declines or remains roughly constant at $z \gtrsim 3$; however, in order to satisfy the Gunn-Peterson constraint for the ionization of the IGM, the SFR needs to rise at high redshifts above its value at $z \sim 3$ (Madau 1999).
The presence of a substantial population of faint quasars at $z \gtrsim 5$ is therefore consistent with current data and is suggested by cosmological models for hierarchical structure formation. If future data would indicate a nonincreasing cosmic SFR at $z \gtrless 3$, then quasars would be the likely sources of reionization. Recent theoretical models have focused on the properties of the quasar population in the optical (Haehnelt \& Rees 1993; Haiman \& Loeb 1998; Haehnelt et al. 1998), and in the infrared (e.g., Sanders 1999; Almaini, Lawrence, \& Boyle 1999). These models were shown to be only mildly constrained by the lack of $z \gtrsim 3.5$ quasars in the Hubble deep field (HDF; see Haiman, Madau, \& Loeb 1999).

In this Letter we point out that quasars can be best distinguished from star-forming galaxies at high redshifts by their $\mathrm{X}$-ray emission. First, we illustrate the agreement between our simplest hierarchical model for quasars and existing ROSAT data on the X-ray LF (Miyaji et al. 1998a) and the soft X-ray background (XRB, Miyaji et al. 1998b). We then show that forthcoming X-ray observations with the Chandra $\mathrm{X}$-ray $\mathrm{Ob}$ servatory ( $C X O$; formerly known as $A X A F)$ will be able to probe the abundance of quasars during the reionization of the universe at $z \gtrsim 5$.

\section{MODEL DESCRIPTION AND QUASAR SPECTRA}

Our model is based on the Press-Schechter mass function of CDM halos (see Haiman \& Loeb 1998 for complete details). We assume that each halo of mass $M_{\text {halo }}$ forms a central black hole of mass $M_{\mathrm{bh}}$. The black hole mass fraction $r \equiv$ $M_{\mathrm{bh}} / M_{\text {halo }}$ is assumed to obey a log-Gaussian probability distribution

$$
p(r)=\exp \left[-\left(\log r-\log r_{0}\right)^{2} / 2 \sigma^{2}\right],
$$

with $\log r_{0}=-3.5$ and $\sigma=0.5$ (Haiman \& Loeb 1999b). These values roughly reflect the distribution of black hole to bulge mass ratios found in a sample of 36 local galaxies (Magorrian et al. 1998) for a baryonic mass fraction of $\sim\left(\Omega_{b} / \Omega_{0}\right) \approx 0.1$. We further postulate that each black hole emits a time-dependent bolometric luminosity in proportion to its 


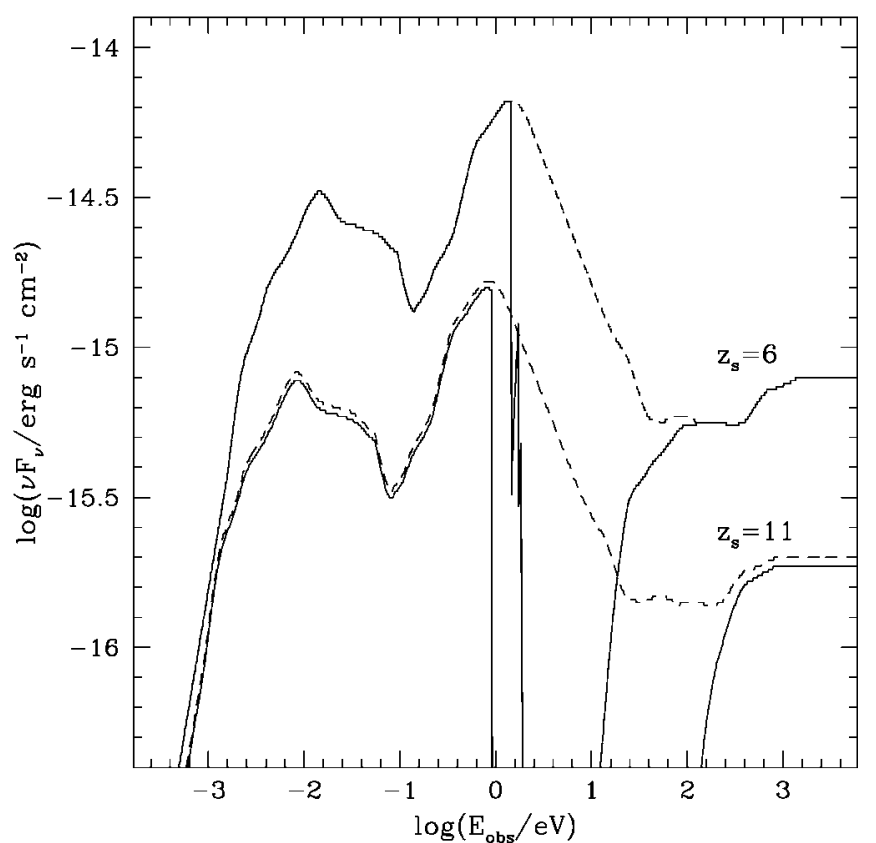

FIG. 1.-Observed spectra of quasars with a central black hole mass of $M_{\mathrm{bh}}=10^{8} M_{\odot}$. The upper curves correspond to a source redshift of $z_{s}=6$ and the lower curves to a source redshift of $z_{s}=11$. In both cases we assume sudden reionization at $z_{r}=10$. The dashed curves show the assumed intrinsic spectral shape, and the solid curves show the spectra after absorption by neutral $\mathrm{H}$ and $\mathrm{He}$.

mass, $L_{q} \equiv M_{\mathrm{bh}} f_{q}=M_{\mathrm{bh}} L_{\mathrm{Edd}} \exp \left(-t / t_{0}\right)$, where $L_{\mathrm{Edd}}=$ $1.5 \times 10^{38} M_{\mathrm{bh}} / M_{\odot} \operatorname{ergs~s}^{-1}$ is the Eddington luminosity, $t$ is the time elapsed since the formation of the black hole, and $t_{0}=10^{6} \mathrm{yr}$ is the characteristic quasar lifetime. Finally, we assume that the shape of the emitted spectrum follows the mean spectrum of the quasar sample in Elvis et al. (1994) up to a photon energy of $10 \mathrm{keV}$. We extrapolate the spectrum up to $\sim 50 \mathrm{keV}$, assuming a spectral slope of $\alpha=0$ (or a photon index of -1$)$.

This simple model was demonstrated to accurately reproduce the evolution of the optical luminosity function in the $B$ band (Pei 1995) at redshifts $z \gtrsim 2.2$ (Haiman \& Loeb 1998). Because our model incorporates several simplifying assumptions, we regard it as the minimal toy model which successfully reproduces the existing data. In this Letter, we focus on the predictions of this minimal model in anticipation of the forthcoming launch of $C X O$; an investigation of a broader range of plausible toy models will be made elsewhere. We adopt the concordance cosmology of Ostriker \& Steinhardt (1995), namely a $\Lambda \mathrm{CDM}$ model with a tilted power spectrum $\left(\Omega_{0}, \Omega_{\Lambda}, \Omega_{b}, h, \sigma_{8 h^{-1}}\right.$, $n)=(0.35,0.65,0.04,0.65,0.87,0.96)$.

Figure 1 shows the adopted spectrum of quasars, assuming a black hole mass $M_{\mathrm{bh}}=10^{8} M_{\odot}$, placed at two different redshifts, $z_{s}=11$ and $z_{s}=6$. In computing the intergalactic absorption, we included the opacity of both hydrogen and helium as well as the effect of electron scattering. We assumed that reionization occurred at $z_{r}=10$ and that at higher redshifts the IGM was homogeneous and fully neutral. At lower redshifts, $0<z<z_{r}$, we included the hydrogen opacity of the Ly $\alpha$ forest given by Madau (1995), and extrapolated his fitting formulae for the evolution of the number density of absorbers beyond $z=5$ when necessary. As Figure 1 shows, the minimum black hole mass detectable by the $\sim 2 \times 10^{-16} \mathrm{ergs} \mathrm{s}^{-1} \mathrm{~cm}^{-2}$ flux limit of $C X O$ (see below) is $M_{\mathrm{bh}} \sim 10^{8} M_{\odot}$ at $z=10$ and $M_{\mathrm{bh}} \sim$

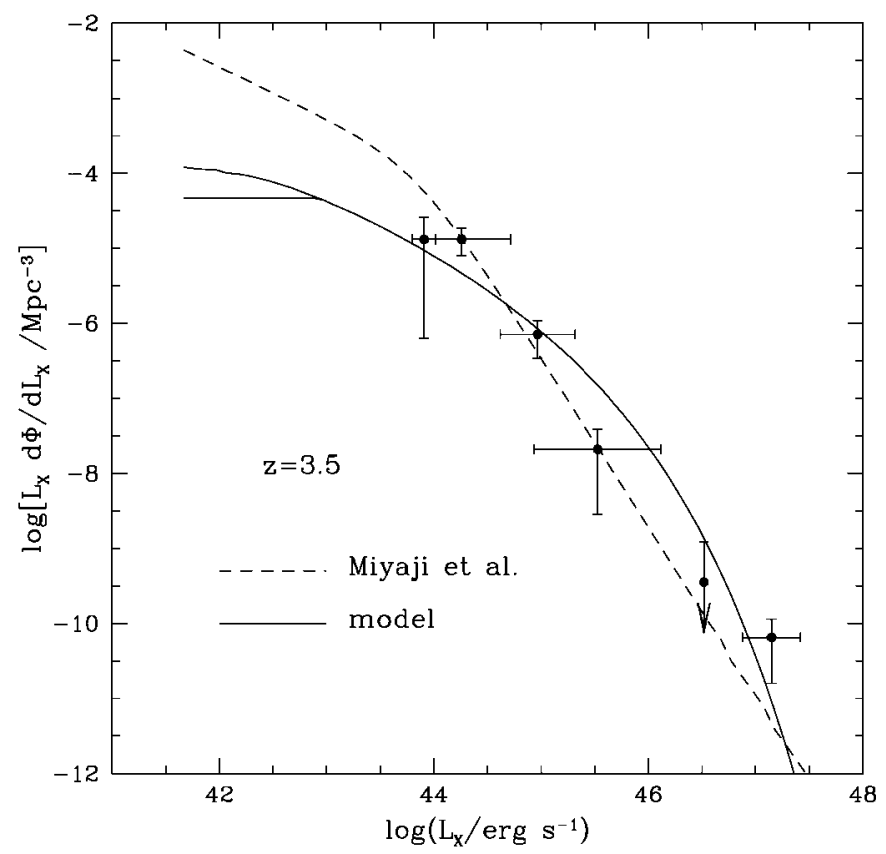

Fig. 2.-Predicted X-ray luminosity function at $z=3.5$ in our model (solid curves). The lower curve shows the effect of a cutoff in circular velocity for the host halos of $v_{\text {circ }} \geq 50 \mathrm{~km} \mathrm{~s}^{-1}$. The ROSAT data points are adopted from Miyaji et al. (1998a), and the dashed curve shows their fitting formula (for our background cosmology).

$2 \times 10^{7} M_{\odot}$ at $z=5$. In our model, the corresponding halo masses are $M_{\text {halo }} \sim 3 \times 10^{11}$, and $M_{\text {halo }} \sim 6 \times 10^{10} M_{\odot}$, respectively. Although such massive halos are rare, their abundance is detectable in wide-field surveys.

\section{THE X-RAY LUMINOSITY FUNCTION}

In our model, the X-ray luminosity function at a redshift $z$ [in $\mathrm{Mpc}^{-3}\left(\operatorname{ergs~s}^{-1}\right)^{-1}$ ] is given by a sum over halos that formed just before that redshift,

$$
\phi\left(L_{\mathrm{X}}, z\right)=\left.\int_{0}^{t(z)} \frac{d t}{f_{\mathrm{X}} f_{q}(\Delta t)} \frac{d^{2} N}{d M_{\mathrm{bh}} d t}\right|_{M_{\mathrm{bh}}=L_{\mathrm{X}} / f_{\mathrm{X}} f_{q}(\Delta t)},
$$

where $L_{\mathrm{X}}$ is the observed X-ray luminosity in the instrument's detection band (0.5-3 keV for ROSAT and 0.4-6 keV for CXO); $f_{\mathrm{X}}$ is the fraction of the quasar's bolometric luminosity emitted in this band; $d^{2} N / d M_{\mathrm{bh}} d t$ is the black halo formation rate, given by a convolution of the Press-Schechter halo mass function with equation (1); and $\Delta t=t(z)-t$ is the time elapsed from a cosmic time $t$ until a redshift $z$.

Although our model was constructed so as to fit the observed optical LF, Figure 2 demonstrates that it is also in good agreement with the data on the X-ray LF. This implies that the choice of quasar spectrum in our model is reasonable. The solid curve in this figure shows the prediction of equation (2) at $z=3.5$, near the highest redshift where X-ray data are available. The bottom curve corresponds to a cutoff in circular velocity for the host halos of $v_{\text {circ }} \geq 50 \mathrm{~km} \mathrm{~s}^{-1}$, which is introduced here in order not to overpredict the number of quasars in the HDF (Haiman et al. 1999). The data points are from recent ROSAT measurements, and the dashed curve in this figure shows a fitting formula from Miyaji et al. (1998a, 1998b). Note that the 


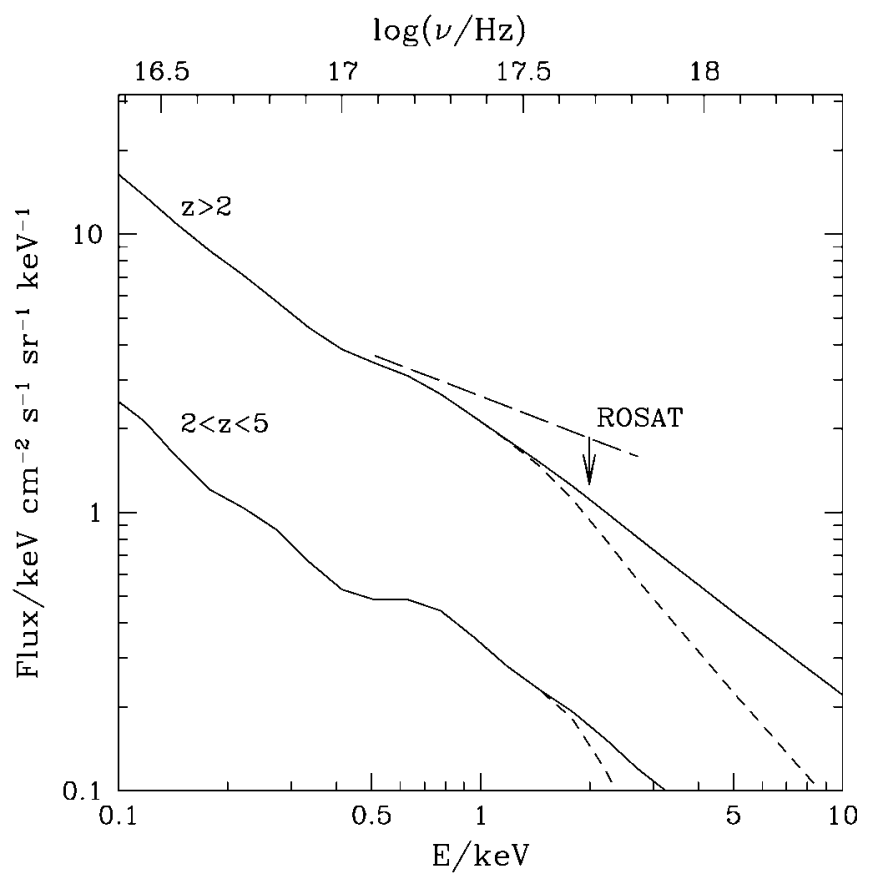

FIG. 3.-Spectrum of the unresolved soft X-ray background in our model. We assume that the median X-ray spectrum of each source follows the mean spectrum of Elvis et al. (1994) up to $10 \mathrm{keV}$ and has a spectral slope of 0.5 (solid lines) or -0.5 (short-dashed lines) at higher photon energies. The lower curves show the spectra resulting from quasars with redshifts between $2<$ $z<5$, and the upper curves include contributions from all redshifts $z>2$. The long-dashed line shows the unresolved fraction (assumed to be 25\%) of the soft X-ray background spectrum from Miyaji et al. (1998b).

faintest quasar actually observed has $L_{\mathrm{X}} \lesssim 10^{44} \mathrm{ergs} \mathrm{s}^{-1}$, and the fitting formula below this luminosity is highly uncertain. Nevertheless, it is important to remember that our model was calibrated so as to fit the observed optical luminosity function of quasars; the existence of a population of obscured quasars which are faint in the optical band but bright in X-rays could increase the number counts beyond our model predictions.

\section{THE X-RAY BACKGROUND}

Existing estimates of the X-ray background (XRB) provide another useful check on our quasar model. The unresolved background flux at a photon energy $E$ is given by (Peebles 1993, p. 125)

$$
F(E)=c \int_{0}^{\infty} d z \frac{d t}{d z} j\left(E_{z}, z\right) \mathrm{keV} \mathrm{cm} \mathrm{cm}^{-1} \mathrm{sr}^{-1} \mathrm{keV}^{-1}
$$

where $E_{z}=E(1+z)$; and $j\left(E_{z}, z\right)$ is the comoving emissivity at a local photon energy $E_{z}$, in units of $\mathrm{keV} \mathrm{cm}^{-3} \mathrm{~s}^{-1} \mathrm{sr}^{-1}$ $\mathrm{keV}^{-1}$, from quasars shining at a redshift $z$. This emissivity is a sum over all quasars whose individual observed flux at $z=0$ is below the ROSAT PSPC detection limit for discrete sources of $2 \times 10^{-15} \mathrm{ergs} \mathrm{cm}^{-2} \mathrm{~s}^{-1}$ (Hasinger \& Zamorani 1999).

Figure 3 shows the predicted spectrum of the XRB in our model at $z=0$ (solid lines). In computing the background spectrum, we ignored $\mathrm{H}$ and $\mathrm{He}$ absorption in the IGM, since its effect is small $(\$ 15 \%)$ near $100 \mathrm{eV}$ and negligibly small above $200 \mathrm{eV}$. We also carried out the integral in equation (3)

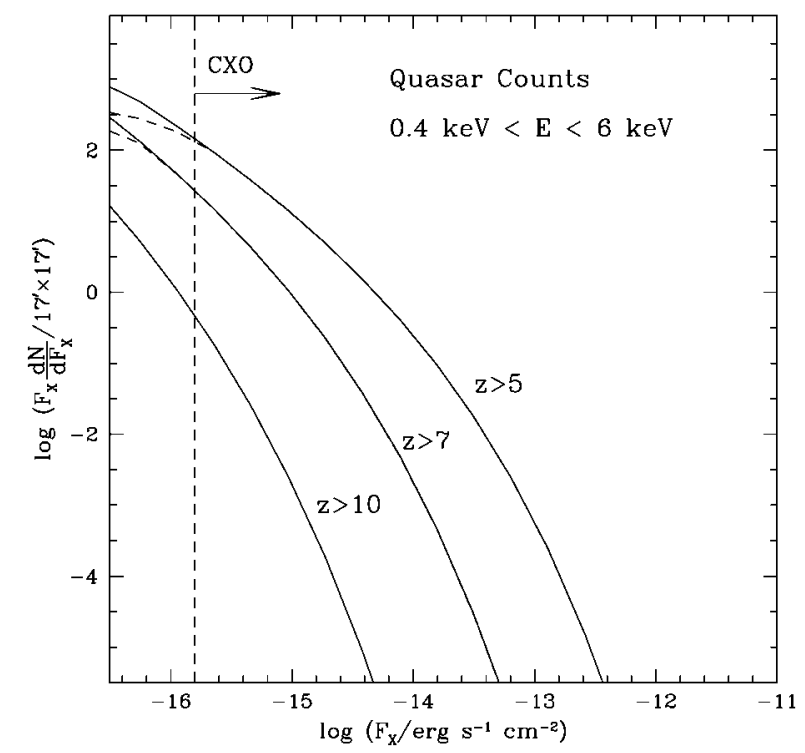

FIG. 4.-Total number of quasars with redshifts exceeding $z=5, z=7$, and $z=10$ are shown as a function of observed X-ray flux in the $C X O$ detection band. The solid curves correspond to a cutoff in circular velocity for the host halos of $v_{\text {circ }} \geq 50 \mathrm{~km} \mathrm{~s}^{-1}$, the dashed curves to a cutoff of $v_{\text {circ }} \geq$ $100 \mathrm{~km} \mathrm{~s}^{-1}$. The vertical dashed line show the CXO sensitivity for a $5 \sigma$ detection of a point source in an integration time of $5 \times 10^{5} \mathrm{~s}$.

only for $z>2$, the redshift range where our model is valid (Haiman \& Loeb 1998). The short-dashed lines show the predicted fluxes assuming a steeper spectral slope beyond $10 \mathrm{keV}$ ( $\alpha=-0.5$, or a photon index of -1.5 ). The long-dashed line shows the $25 \%$ unresolved fraction of the soft XRB observed with ROSAT (Miyaji et al. 1998b; Fabian \& Barcons 1992). This fraction represents the observational upper limit on the component of the soft XRB that could in principle arise from high-redshift quasars. As the figure shows, our quasar model predicts an unresolved flux just below this limit in the 0.5-3 $\mathrm{keV}$ range. The model also predicts that most ( $\gtrsim 90 \%)$ of this yet unresolved fraction arises from quasars beyond $z=5$.

\section{PREDICTED NUMBER COUNTS FOR THE CHANDRA X-RAY OBSERVATORY}

By summing the luminosity function over redshifts, we obtain the number counts of quasars per solid angle expected to be detectable in a flux interval $d F_{\mathrm{X}}$ around $F_{\mathrm{X}}=L_{\mathrm{X}} / 4 \pi d_{L}^{2}(z)$, for all sources above a redshift $z$ :

$$
\frac{d N}{d F_{\mathrm{X}} d \Omega}=\int_{z}^{\infty} d z\left(\frac{d V}{d z d \Omega}\right) \phi\left(L_{\mathrm{X}}, z\right) 4 \pi d_{L}^{2}(z)
$$

where $(d V / d z d \Omega)$ is the comoving volume element per unit redshift and solid angle, and $d_{L}(z)$ is the luminosity distance at a redshift $z$.

In Figure 4, we show the predicted counts from equation (4) in the $0.4-6 \mathrm{keV}$ energy band of the CCD Imaging Spectrometer (ACIS) of $C X O$. Note that these curves are insensitive to our extrapolation of the template spectrum beyond $10 \mathrm{keV}$. The figure is normalized to the $17^{\prime} \times 17^{\prime}$ field of view of the imaging chips. The solid curves show that of order a hundred quasars with $z>5$ are expected per field at the $C X O$ sensitivity of $\sim 2 \times 10^{-16} \mathrm{ergs} \mathrm{s}^{-1} \mathrm{~cm}^{-2}$ for a $5 \sigma$ detection of a point 
source. Note that $C X O$ 's arcsecond resolution will ease the separation of these point sources from background noise. The abundance of quasars at higher redshifts declines rapidly; however, a few objects per field are still detectable at $z \sim 8$. The dashed lines show the results for a minimum circular velocity of the host halos of $v_{\text {circ }} \geq 100 \mathrm{~km} \mathrm{~s}^{-1}$ and imply that the model predictions for the $C X O$ satellite are not sensitive to such a change in the host velocity cutoff. This is because the halos shining at the $C X O$ detection threshold are relatively massive, $M_{\text {halo }} \sim 10^{11} M_{\odot}$, and possess a circular velocity above the cutoff. In principle, the number of predicted sources would be lower if we had assumed a steeper spectral slope. For example, as Figure 3 shows, our model falls short of predicting the hard $\mathrm{X}$-ray background, by about an order of magnitude at $10 \mathrm{keV}$. The difference could be explained by a change in our template spectrum to include a population of quasars with hard, but highly absorbed spectra (caused by the denser, and more gas rich hosts at high redshift). We note, however, that the agreement between the LF predicted by our model at $z \approx 3.5$ and that inferred from ROSAT observations would be upset by such a change and require a modification of the model that would in turn tend to counterbalance the decrease in the predicted counts.

\section{DISCUSSION}

The existence of quasars at redshifts $z \gtrsim 5$ has important consequences for the reionization history of the universe. Quasars can be easily distinguished from stellar systems by their $\mathrm{X}$-ray emission. We have demonstrated that state-of-the-art Xray observations could provide more stringent constraints on quasar models than currently provided by the Hubble deep field (Haiman et al. 1999). In particular, we have found that forthcoming X-ray observations with the $C X O$ satellite might detect of order a hundred quasars per field of view in the redshift interval $5 \lesssim z \lesssim 8$. Our numerical estimates are based on the simplest toy model for quasar formation in a hierarchical CDM cosmology that satisfies all the current observational constraints on the optical and X-ray luminosity functions of quasars. Al- though a more detailed analysis is needed in order to assess the modeling uncertainties in our predictions, the importance of related observational programs with $C X O$ is evident already from the present analysis. Other future instruments, such as the HRC or the ACIS-S cameras on $C X O$, or the EPIC camera on $X M M$, might also be useful in searching for high-redshift quasars.

Follow-up optical and infrared observations are needed in order to identify the redshifts of the faint pointlike sources that might be detected by the $C X O$ satellite. Quasars emit a broad spectrum which extends into the UV and includes strong emission lines, such as Ly $\alpha$. For quasars near the $C X O$ detection threshold, the fluxes at $\sim 1 \mu \mathrm{m}$ are expected to be relatively high, $\sim 0.5-0.8 \mu \mathrm{Jy}$. Therefore, infrared spectroscopy of X-ray selected quasars could prove to be a particularly useful approach for unraveling the reionization history of the intergalactic medium at $z \gtrsim 5$ (for the potential lessons to be learned, see Miralda-Escudé 1998, Haiman \& Loeb 1999a, Loeb \& Rybicki 1999, and Rybicki \& Loeb 1999).

At present, the best constraints on hierarchical models of the formation and evolution of quasars originate from the Hubble deep field. However, HST observations are only sensitive to a limiting magnitude of $V \sim 29$ and cannot probe the earliest quasars. The Next Generation Space Telescope (NGST), scheduled for launch in 2008, will achieve nJy sensitivity at wavelengths $1-3 \mu \mathrm{m}$ and could directly probe the earliest quasars (Haiman \& Loeb 1998). The combination of X-ray data from the $C X O$ satellite and infrared spectroscopy from NGST could potentially resolve one of the most important open questions about the thermal history of the universe, namely whether the intergalactic medium was reionized by stars or by accreting black holes.

We thank G. Hasinger and R. Mushotzky for discussions that motivated this study. We also thank N. White and M. Elvis for useful comments and T. Miyaji for supplying the data in Figure 2. Z. H. was supported by the DOE and the NASA grant NAG5-7092 at Fermilab. A. L. was supported in part by NASA grants NAG5-7039 and NAG5-7768.

\section{REFERENCES}

Almaini, O., Lawrence, A., \& Boyle, B. J. 1999, MNRAS, in press (astro-ph/ 9903178)

Barger, A. J., Cowie, L. L., \& Sanders, D. B. 1999, ApJ, 518, L5

Chen, H.-W., Lanzetta, K., \& Pascarelle, S. 1999, Nature, 398, 586

Elvis, M., et al. 1994, ApJS, 95, 1

Fabian, A. C., \& Barcons, X. 1992, ARA\&A, 30, 429

Fan, X., et al. (SDSS collaboration) 1999, AJ, in press (astro-ph/9903237)

Haehnelt, M. G., Natarajan, P., \& Rees, M. J. 1998, MNRAS, 300, 817

Haehnelt, M. G., \& Rees, M. J. 1993, MNRAS, 263, 168

Haiman, Z., \& Loeb, A. 1998, ApJ, 503, 505

$$
\text { 1999a, ApJ, 519, } 479
$$

1999b, in Proc. Ninth Annual October Astrophysics Conference in Maryland, After the Dark Ages: When Galaxies Were Young (The Universe at $2<z<5$ ), ed. S. Holt \& E. Smith (New York: AIP), 34

Haiman, Z., Madau, P., \& Loeb, A. 1999, ApJ, 514

Hasinger, G., \& Zamorani, G. 1999, in Festschrift for R. Giacconi's 65th Birthday, ed. H. Gursky, R. Ruffini, L. Stella (Singapore: World Scientific), in press (astro-ph/9712341)

Loeb, A., \& Rybicki, G. 1999, ApJ, in press (astro-ph/9902180)

Madau, P. 1995, ApJ, 441, 18
Madau, P. 1999, in Phys. Scripta, in press (astro-ph/9902228)

Magorrian, J., et al. 1998, AJ, 115, 2285

Miralda-Escudé, J. 1998, ApJ, 501, 15

Miyaji, T., Hasinger, G., \& Schmidt, M. 1998a, in Highlights in X-Ray Astronomy, in press (astro-ph/9809398)

Miyaji, T., Ishisaki, Y., Ogasaka, Y., Ueda Y., Freyberg, M. J., Hasinger, G., \& Tanaka, Y. 1998b, A\&A 334, L13

Ostriker, J. P., \& Steinhardt, P. J. 1995, Nature, 377, 600

Peebles, P. J. E. 1993, Principles of Physical Cosmology (Princeton: Princeton Univ. Press)

Pei, Y. C. 1995, ApJ, 438, 623

Press, W. H., \& Schechter, P. L. 1974, ApJ, 181, 425

Rybicki, G., \& Loeb, A. 1999, ApJL, submitted (astro-ph/9903291)

Sanders, D. B. 1999, in Proc. IAU Symp. 194, Activity in Galaxies and Related Phenomena (San Francisco: ASP), in press (astro-ph/9903445)

Schmidt, M., Schneider, D. P., \& Gunn, J. E. 1995, AJ, 110, 68

Shaver, P. A., et al. 1996, Nature, 384, 439

Weymann, R. J., Stern, D., Bunker, A., Spinrad, H., Chaffee, F. H., Thompson, R. I., \& Storrie-Lombardi, L. J. 1998, ApJ, 505, 95 